\title{
Spatio-temporal SIFT and Its Application to Human Action Classification
}

\author{
Manal Al Ghamdi ${ }^{1}$, Lei Zhang ${ }^{2}$, and Yoshihiko Gotoh ${ }^{1}$ \\ 1 University of Sheffield, UK \\ 2 Harbin Engineering University, PRC
}

\begin{abstract}
This paper presents a space-time extension of scale-invariant feature transform (SIFT) originally applied to the 2-dimensional (2D) volumetric images. Most of the previous extensions dealt with 3dimensional (3D) spacial information using a combination of a 2D detector and a 3D descriptor for applications such as medical image analysis. In this work we build a spatio-temporal difference-of-Gaussian (DoG) pyramid to detect the local extrema, aiming at processing video streams. Interest points are extracted not only from the spatial plane $(x y)$ but also from the planes along the time axis $(x t$ and $y t)$. The space-time extension was evaluated using the human action classification task. Experiments with the KTH and the UCF sports datasets show that the approach was able to produce results comparable to the state-of-the-arts.
\end{abstract}

\section{Introduction}

Consider a task of detecting humans and their motions in a video stream. It is a challenging task and requires identification of different levels of features that represent human presence and activities. It has been shown that a number of interest point based approaches can deal with this task [1-3]. They typically used a bag-of-features (BoF) model for feature representation, and proved their robustness to location changes and to noises. However, they depended mainly on the descriptor phase to produce discriminative representation of a video, discarding information relevant to the distribution of interest points in the spatio-temporal domain. As a consequence, the features produced often lacked temporal information for describing smooth motions. Furthermore, they were not able to address the scale and location invariance in the temporal domain.

Since its original development by Lowe [4], scale invariant feature transform (SIFT) has been successful in various image processing applications for locally detecting and describing interest points. It has proved its efficiency with tasks in a 2D space such as image similarity and classification. Recently its extension to higher dimensional spaces has been explored in order to represent more complex data. In this paper we present a SIFT extension for detecting interest points that can have significant local variations in both the spatial and temporal domains. Identified features are invariant to scale, location and orientation changes. The work is related to two recent studies: firstly Dorr et al. constructed spatiotemporal pyramids as a multi-resolution representation for video streams [5].

A. Fusiello et al. (Eds.): ECCV 2012 Ws/Demos, Part I, LNCS 7583, pp. 301-310, 2012.

(C) Springer-Verlag Berlin Heidelberg 2012 
They applied these pyramids to visualise dynamic gaze density maps. The second one was presented by Lopes et al. for human action recognition application 6]. They collected 2D SIFT and 2D SURF (speeded up robust features) interest points on the $x y$ plane along the spatial domain and on the $x t$ and $y t$ planes along the spatio-temporal domain.

The approach consists of two stages: transformation of $3 \mathrm{D}$ (2D space and time) video signal to spatio-temporal pyramids, followed by extraction of interest points from the spacial and the spatio-temporal planes. The spatio-temporal SIFT (ST-SIFT) detector is presented in Section 3, To describe the region around the detected points we use the 3D SIFT descriptor developed by [7] that calculates the spatio-temporal gradient for each pixel in the given cuboid. The approach leads to local regions that are invariant to scale and location in both the spatial and the temporal domains. The contribution of this paper can be summarised as follows:

* Construction of multi-resolution space-time Gaussians and difference-ofGaussian (DoG) pyramids, where each level contains a 3D smoothed and subsampled version of the previous level;

* Provision of an interest point detection schema from three different planes along the spatial and the temporal axes;

* Formulation of the space-time detector that is scale and location invariant;

* Application of the developed ST-SIFT on a human action classification task, with comparison to other state-of-the-art approaches.

\section{Related Work}

Recently, local features have received a great deal of attention in video-processing applications. They are extended to take into account the spatio-temporal nature of video data. Laptev and Lindeberg [8] extended the well-known Harris-Laplace detector in the spatial domain to the spatio-temporal domain. Schuldt et al. presented a video representation based on detected interest points combined with a support vector machine (SVM) classification schema for action recognition [3]. Motivated by these studies, Dollar et al. developed an approach to behaviour recognition, extracting local maxima from the space and the temporal domains based on responses of the Gaussian filter convolved with a pair of 1D Gabor filters [1].

SIFT extension can be categorised into three groups: (1) extension of the descriptor part only, combined with 2D detectors, (2) a full 3D spatial extension, and (3) a combination of different approaches to separately describing motion and appearance. One example of the first category is by Scovanner et al., who extended the descriptor to the time domain and dropped the scale and location invariance covered by the detector 77]. In the second category Cheung and Hamarneh generalised SIFT to $n$-dimensional space $\left(n\right.$-SIFT) with $2^{5 n-3}$ dimensional features vector 9]. Allaire et al. also developed a full 3D extension, addressing two important issues not previously solved, namely, extracted points with low contrast and the full 3D orientation invariance [10]. Unlike the other 
groups Chen and Hauptmann handled the spatial and the temporal domains separately [11]. Their MoSIFT descriptor contained two parts: describing the spatial domain with a histogram of gradient (HOG) and the temporal domain with a histogram of optical flow (HOF) that captured moves of interest points.

\section{Spatio-temporal SIFT Detector}

\subsection{Conventional 2D SIFT Detector}

The 2D SIFT detector maps the spacial content of an image to a coordinate of scale, location and orientation invariant feature. This is achieved using a scalespace kernel function such as the Gaussian, which is a continuous function to capture stable features in different scales. The Gaussian function on a point $(x, y)$ at scale $\sigma$ can be defined as

$$
G(x, y, \sigma)=\frac{1}{2 \pi \sigma^{2}} \exp \left(-\frac{x^{2}+y^{2}}{2 \sigma^{2}}\right)
$$

The scale-space function $L(x, y, \sigma)$ for the input image $I(x, y, \sigma)$ can be

$$
L(x, y, \sigma)=G(x, y, \sigma) * I(x, y, \sigma)
$$

where $*$ is the convolution operation. The following $D(x, y, \sigma)$ indicates stable locations in the scale-space, derived by convolution of the input image with the DoG functions:

$$
\begin{aligned}
D(x, y, \sigma) & =(G(x, y, K \sigma)-G(x, y, \sigma)) * I(x, y, \sigma) \\
& =L(x, y, K \sigma)-L(x, y, \sigma)
\end{aligned}
$$

where the DoG function is the difference between two neighbours with the constant scale factor $K$. Finally the maxima and the minima of $D(x, y, \sigma)$ give scale-invariant points in the scale-space.

\subsection{Spatio-temporal Difference of Gaussian Pyramid}

To achieve the invariance in both space and time, we first calculate a spatiotemporal DoG pyramid. Interest points are extracted in three different planes - $x y, y t$, and $x t$ - from the DoG. Points that are common in three planes carry vital information in both the spatial and the temporal domains. The DoG pyramid is a bandpass version of the original signal, which serves a scale-space of video to detect invariant interest points [5]. Unlike the previous works for SIFT extension where they constructed 3D spatial pyramids, we treat both the spatial and the temporal domains equally. The spatio-temporal Gaussian pyramid was originally introduced by $\mathrm{Uz}$ et al. in 1991, where downsampling was performed separately in the spatial and the temporal domains [12]. This means that every lower level in the pyramid is generated by dropping every other pixel in the spatial domain followed by dropping every other frame in the temporal domain. 

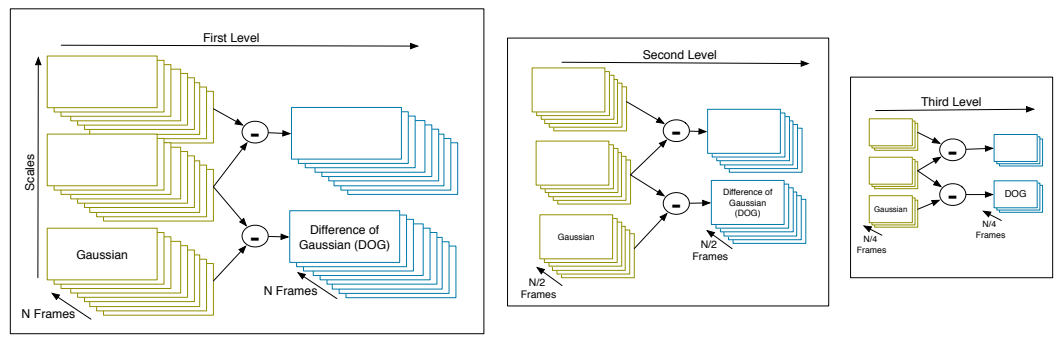

Fig. 1. Video pyramids. Each level is spatially and temporally downsampled from the previous level, and convolution with the 3D Gaussian is calculated to create a Gaussian pyramid. The DoG is then constructed by subtracting the adjacent Gaussian scales.

For a video sequence with a frame size of $W \times H$, let $I(x, y, t)$ denote a pixel at location $(x, y)$ in frame $t$. We construct the Gaussian pyramid of $N$ levels where $N$ is determined by the frame size. $G_{i}(i=0, \ldots, N-1)$ represents each level of the pyramid, where the highest level $G_{0}(t)$ corresponds to the original video frame sequence. This process leads to the multi-level spatio-temporal Gaussians and the DoG pyramids shown in Figure 1, Incremental convolution of video signal $I$ with the 3D Gaussian filter $G$ results in the scale space $L$ of the first level:

$$
L(x, y, t, \sigma, \tau)=G(x, y, t, \sigma, \tau) * I(x, y, t, \sigma, \tau)
$$

with multiple scales $S$ separated by a constant value of $K=2^{1 / S}$. The spatiotemporal Gaussian function with the spatial and the temporal scale parameters, $\sigma$ and $\tau$, is given by

$$
G(x, y, t, \sigma, \tau)=\frac{1}{(2 \pi)^{\frac{3}{2}} \sigma^{2} \tau} \exp \left(-\frac{x^{2}+y^{2}}{2 \sigma^{2}}-\frac{t^{2}}{2 \tau^{2}}\right)
$$

Following Lowe [4], $S+3$ scales are generated for each level to guarantee that local extrema detection will cover the complete octave. To produce a lower level the signal is spatially and temporally downsampled with the Gaussian at scales $\sigma$ and $\tau$. This yields a level with the lower frame rate and frames of the smaller size (illustrated on the left side in each box of Figure 1). The frame size at level $G_{i}$ is $W / 2^{i} \times H / 2^{i}$, and $G_{i}(t)$ matches $G_{0}\left(2^{i} t\right)$ at time $t$ (see this in Figure 2). The next step is to construct a DoG pyramid; for each level in the Gaussian pyramid, a DoG of one lower octave is derived by subtracting the Gaussian of the adjacent scales:

$$
\begin{aligned}
D(x, y, \sigma, \tau) & =(G(x, y, K \sigma, \tau)-G(x, y, \sigma, \tau)) * I(x, y, \sigma, \tau) \\
& =L(x, y, K \sigma, \tau)-L(x, y, \sigma, \tau)
\end{aligned}
$$

This is shown on the right side in each box of Figure 1 


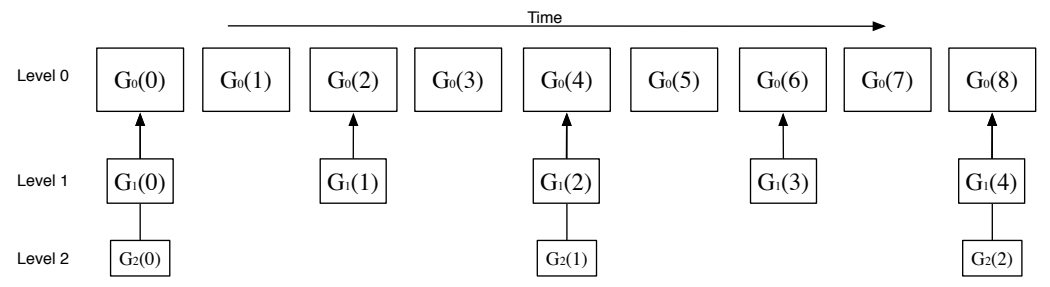

Fig. 2. Mapping strategy between three levels in the spatio-temporal Gaussian pyramid. A pixel $G_{i}(t)$ at level $G_{i}$ maps to a pixel $G_{0}\left(2^{i} t\right)$. For example, $G_{1}(3)$ mapped to $G_{0}(6)$ and $G_{2}(1)$ mapped to $G_{0}(4)$.

\subsection{Interest Points Detection}

Once the DoG pyramid is constructed, local extrema of the adjacent scales in the $x y, x t$ and $y t$ planes are compared. The assumption here is that spatio-temporal events can be described by common interest points between the spatial axis (appearance information) and the temporal axis (motion information). Lopes et al. presented an approach to forming a spatio-temporal volume by stacking a set of frames from a video signal [6]. There are three directions to slice this volume into planes, as illustrated in Figure 3. One can slice through the spatial axis to create $x y$ planes. Alternatively one can create a sequence of planes from the temporal axis combined with either the $x$ or $y$ spatial axis.

Extrema are detected from each slice of the spatio-temporal pyramid separately, and the union of common extrema in three directions are selected as interest points. It may be required to introduce some tolerance controlled by some threshold. This is because identified extrema may not be a pixel at exactly the same position although close to each other. Similar to the original 2D SIFT by Lowe [4], local extrema are detected by comparing each sample point to its eight neighbours in the current scale and the nine neighbours in the scales above and below. This is performed at each level within the DoG pyramid. In the end

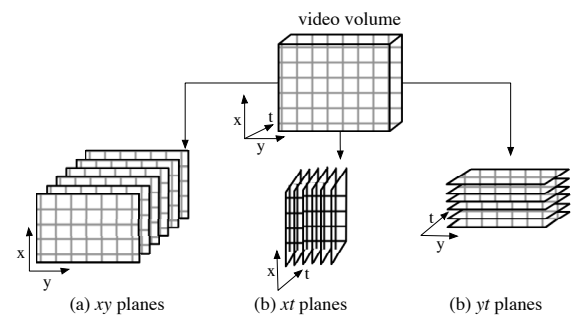

Fig. 3. The video volume and the generated planes along different directions. Three axes of each DoG scale volume represent $x$ and $y$ in the spacial and $t$ in the temporal dimensions, creating three planes, $x y, x t$, and $y t$. 


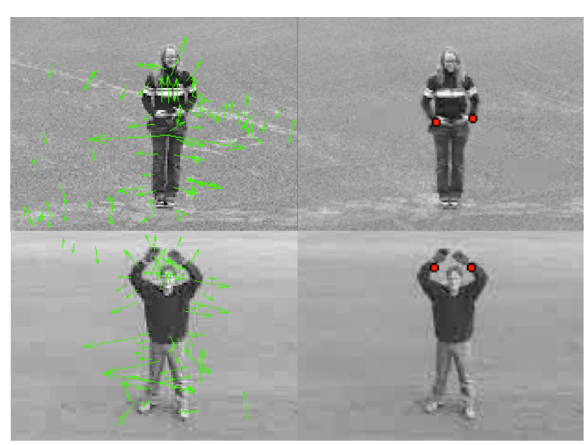

Fig. 4. Comparison between the interest points extracted with the 2D-SIFT (left) and the proposed ST-SIFT (right). The 2D-SIFT defines the spatial points only from the moving objects and the background, while the ST-SIFT defines the spatial points from different scales that have motion information.

filtering may be applied to remove noisy points and edges. Figure 4 shows the difference between the interest points extracted, from the KTH hand-waving action, using the traditional 2D-SIFT and the proposed ST-SIFT.

\section{Experiments}

We evaluated the ST-SIFT detector using the human action classification task.

\subsection{Implementation}

To extract interest points from the spatio-temporal video cube, ST-SIFT was built on a 2D-SIFT based image classifier in the VLFeat toolbox [13]. A BoF model implemented in this toolbox was an open library containing various algorithms for computer vision applications. The spatio-temporal regions around the interest points were described by the 3D HOG [7]. Publicly available code by Scovanner et al. was used, which is slightly different from what was described in 7]. The descriptor length was 640-dimensional for each interest point, which was determined by the number of bins to represent angles, $\theta$ and $\phi$, in the subhistograms.

The next was the vocabulary learning; descriptors generated for interest points were clustered to a pre-specified number of visual words. We used Elkan's kmeans clustering algorithm, which was faster than the standard Lloyd's k-means. Centres of the generated clusters were referred to as 'visual words' while the set of these words was known as the 'spatio-temporal word vocabulary'. Based on the vocabulary, a frequency histogram was created where visual descriptors were mapped to the visual words. The word frequency in each video was accumulated onto a histogram known as a signature. A support vector machine (SVM) classifier was used to learn a model from signatures for each action. We used a non-linear SVM with a $\chi^{2}$-kernel. 
Table 1. Confusion matrix for the KTH dataset

\begin{tabular}{|c|c|c|c|c|c|c|}
\hline Walk & 100 & 0 & 0 & 0 & $\overline{0}$ & 0 \\
\hline Jog & 0 & 78 & 11 & 0 & 0 & 11 \\
\hline Run & 0 & 11 & 89 & 0 & 0 & 0 \\
\hline Box & 0 & 0 & 0 & 100 & 0 & 0 \\
\hline Wave & 0 & 0 & 0 & 0 & 100 & 0 \\
\hline Clap & 0 & 0 & 11 & 0 & 11 & 78 \\
\hline
\end{tabular}

\subsection{Experimental Setup}

Two publicly available human actions datasets were employed. The first was known as the KTH dataset developed by Schuldt et al. [3]. It contained six different human activities — walking, jogging, running, hand-waving, boxing and hand-clapping. Each action was performed by 25 persons in 4 different scenarios with monotone background. Following the studies conducted by [2] and [14], we divided the dataset into two parts, 16 persons for training and 9 persons for testing.

The second one was the UCF sports dataset, more realistic but challenging data collected from broadcast sport videos by Ahmed et al. [15]. There were nine actions in the publicly available part of this dataset, consisting of diving, golf swinging, kicking, lifting, horseback riding, running, skating, swinging, walking 1 . Following the original paper [15], we used a leave-one-out cross validation training method.

When constructing a Gaussian pyramid, the number of scales was set to three for each of four levels in the KTH dataset, and three for each of three levels in the UCF sports dataset. The codebook size was a key parameter for BoF models. We followed the experiment procedure in [14], and the best performance was obtained for both datasets with the codebook size of 1500 words. A single SVM classifier was built for each action using all training samples.

\subsection{Results}

This section presents the performance of the ST-SIFT interest points detector using two datasets. Tables 1 and 2 show confusion matrices for human action classification experiments with the KTH and the UCF sports datasets. The overall accuracy of each was $90.74 \%$ and $80.56 \%$, respectively. The approach using the ST-SIFT detector was able to clearly distinguish between similar actions such as walking and running.

In the second set of experiments, ST-SIFT was compared with two conventional SIFT algorithms. One was the combination of the original 2D DoG detector and the 2D HOG descriptor by Lowe [4], with which each frame was

${ }^{1}$ The tenth action, pole vaulting, was not publicly available. 
Table 2. Confusion matrix for the UCF sports dataset

\begin{tabular}{|c|c|c|c|c|c|c|c|c|c|}
\hline Dive & 75 & 25 & 0 & 0 & 0 & 0 & $\overline{0}$ & 0 & $\overline{0}$ \\
\hline Golf & 0 & 75 & 25 & 0 & 0 & 0 & 0 & 0 & 0 \\
\hline Kick & 0 & 0 & 100 & 0 & 0 & 0 & 0 & 0 & 0 \\
\hline Lift & 0 & 0 & 0 & 100 & 0 & 0 & 0 & 0 & 0 \\
\hline Rid & 0 & 0 & 0 & 25 & 50 & 25 & 0 & 0 & 0 \\
\hline Run & 0 & 0 & 0 & 0 & 0 & 100 & 0 & 0 & 0 \\
\hline Skate & 0 & 0 & 25 & 0 & 0 & 25 & 50 & 0 & 0 \\
\hline Swing & 0 & 0 & 0 & 0 & 0 & 0 & 25 & 75 & 0 \\
\hline Walk & 0 & 0 & 0 & 0 & 0 & 0 & 0 & 0 & 100 \\
\hline
\end{tabular}

represented separately. Another representation consisted of the 2D DoG detector and the 3D HOG descriptor developed in [7]. Table 3 shows that the ST-SIFT detector followed by the 3D HOG descriptor outperformed the other two representations. This indicates that ST-SIFT is able to (1) capture the interest points that have vital information in both the spatial and the temporal domains, which were missed by the conventional approaches, and to (2) represent events in real video sequences.

\subsection{Comparison of ST-SIFT with the Recent State-of-the-Art}

We compare the performance of ST-SIFT with approaches to interest points extraction published recently using KTH and UCF sports data. Note that some groups applied pre-processing and object tracking steps before extracting interest points while other groups combined multiple techniques to improve the performance. The purpose of this comparison is to show the rough position of ST-SIFT among the recent state-of-the-art techniques in the context of action classification task. ST-SIFT was not the best but among the state-of-the-art in the field.

The KTH dataset has been experimented with various techniques for action recognition task. In 2004, Schuldt et al. [3] combined Laptev [8] local space-time features with spatio-temporal jets to recognising complex motion patterns. Their experiment reported a 71.7\% accuracy. In 2005, Dollar et al. 1] proposed sparse spatio-temporal features extended from the 2D corner detector and described the interest points region using the spatio-temporal cuboid and achieved a recognition accuracy of $81.2 \%$. Laptev et al. [16] improved the accuracy to $91.8 \%$ by describing interest points with the histograms of oriented gradient ( $\mathrm{HoG}$ ) and

Table 3. Comparison of ST-SIFT and conventional detectors

\begin{tabular}{cccc}
\hline detector & descriptor & KTH & UCF \\
\hline ST SIFT & 3D HOG & $90.74 \%$ & $80.56 \%$ \\
2D DoG & 3D HOG & $77.00 \%$ & $77.78 \%$ \\
2D DoG & 2D HOG & $72.22 \%$ & $58.52 \%$ \\
\hline
\end{tabular}


the optic flow $(\mathrm{HoF})$. Niebles et al. 2] on the other hand achieved a lower performance of $83.3 \%$ than Dollar et al. [1] by describing the Dollar interest points with a spatial-temporal gradient cube.

For the UCF sports dataset, Rodriguez et al. [15] achieved $69.2 \%$ accuracy in 2008 by extending the traditional Maximum Average Correlation Height $(\mathrm{MACH})$ filter to 3D volume and combining it with Spatio-temporal Regularity Flow (SPREF). In 2009, Liu et al. [17] reported a recognition rate of $74.5 \%$ by combining different detectors including Harris-Laplacian (HAR), HessianLaplacian (HES) and MSER and then they described the region of interest using Dollar et al. [1]. Wang et al. [18] outperformed them with $85.6 \%$ by dense sampling to extract video blocks and a 3D-HOG descriptor. Recently, Kläser et al. boosted the recognition accuracy to $86.7 \%$ by employing object localisation in the bag-of-visual-features representation [19]. An even better result of $87.3 \%$ accuracy was reached by Kovashka et al. by learning the space-time neighbourhoods in a BoF representation [20].

\section{Conclusion}

In this paper we presented a spatio-temporal extension to the 2D SIFT approach and demonstrated its performance using the task of human action classification. We combined the ST-SIFT detector with the 3D-HOG descriptor and applied to the KTH and the UCF sports datasets. The results showed that ST-SIFT was able detect local features for human activities. The purpose of this development was to extract local features that were invariant to location, scale, orientation and temporal changes. The KTH and the UCF sports datasets did not involve significant scaling and orientation changes; however, ST-SIFT should be able to deliver better performance when such changes are observed. They have been chosen to build a comparison level with the existing approaches that are usually tested on these datasets for this task. Future work includes application to video searching and retrieval using video data with high variation in scale, location and rotation.

Acknowledgements. The first author would like to thank Umm Al-Qura University, Makkah, Saudi Arabia for funding this work as part of her PhD scholarship program. This work is partly sponsored by National Natural Science Foundation of China \#60702053, and Young Teacher Supporting Plan by Harbin Engineering University and Heilongjiang Province, China \#1155G17.

\section{References}

1. Dollar, P., Rabaud, V., Cottrell, G., Belongie, S.: Behavior recognition via sparse spatio-temporal features. In: IEEE International Workshop on Visual Surveillance and Performance Evaluation of Tracking and Surveillance (2005)

2. Niebles, J., Wang, H., Fei-Fei, L.: Unsupervised learning of human action categories using spatial-temporal words. International Journal of Computer Vision 79 (2008) 
3. Schuldt, C., Laptev, I., Caputo, B.: Recognizing human actions: a local SVM approach. In: International Conference on Pattern Recognition, vol. 3 (2004)

4. Lowe, D.G.: Distinctive image features from scale-invariant keypoints. International Journal of Computer Vision 60 (2004)

5. Dorr, M., Jarodzka, H., Barth, E.: Space-variant spatio-temporal filtering of video for gaze visualization and perceptual learning. In: Symposium on Eye-Tracking Research \& Applications, New York (2010)

6. Lopes, A., Oliveira, R., de Almeida, J., de Araujo, A.A.: Spatio-temporal frames in a bag-of-visual-features approach for human actions recognition. In: Brazilian Symposium on Computer Graphics and Image Processing (2009)

7. Scovanner, P., Ali, S., Shah, M.: A 3-dimensional sift descriptor and its application to action recognition. In: International Conference on Multimedia (2007)

8. Laptev, I., Lindeberg, T.: Space-time interest points. In: International Conference on Computer Vision (2003)

9. Cheung, W., Hamarneh, G.: N-sift: N-dimensional scale invariant feature transform for matching medical images. In: International Symposium on Biomedical Imaging: From Nano to Macro (2007)

10. Allaire, S., Kim, J., Breen, S., Jaffray, D., Pekar, V.: Full orientation invariance and improved feature selectivity of $3 \mathrm{~d}$ sift with application to medical image analysis. In: Computer Vision and Pattern Recognition Workshops (2008)

11. Chen, M.Y., Hauptmann, A.: Mosift: Recognizing human actions in surveillance videos. Transform (2009)

12. Uz, K., Vetterli, M., LeGall, D.: Interpolative multiresolution coding of advance television with compatible subchannels. IEEE Transactions on Circuits and Systems for Video Technology 1 (1991)

13. Vedaldi, A., Fulkerson, B.: Vlfeat: an open and portable library of computer vision algorithms. In: International Conference on Multimedia, New York (2010)

14. Shao, L., Mattivi, R.: Feature detector and descriptor evaluation in human action recognition. In: International Conference on Image and Video Retrieval (2010)

15. Rodriguez, M., Ahmed, J., Shah, M.: Action mach a spatio-temporal maximum average correlation height filter for action recognition. In: Conference on Computer Vision and Pattern Recognition (2008)

16. Laptev, I., Marszalek, M., Schmid, C., Rozenfeld, B.: Learning realistic human actions from movies. In: Conference on Computer Vision and Pattern Recognition (2008)

17. Liu, J., Luo, J., Shah, M.: Action recognition in unconstrained amateur videos. In: International Conference on Acoustics, Speech and Signal Processing (2009)

18. Wang, H., Ullah, M.M., Kläser, A., Laptev, I., Schmid, C.: Evaluation of local spatio-temporal features for action recognition. In: British Machine Vision Conference (2009)

19. Kläser, A., Marszałek, M., Laptev, I., Schmid, C.: Will person detection help bagof-features action recognition? Technical Report RR-7373, INRIA Grenoble, France (2010)

20. Kovashka, A., Grauman, K.: Learning a hierarchy of discriminative space-time neighborhood features for human action recognition. In: Conference on Computer Vision and Pattern Recognition (2010) 